\title{
Emergent Phenomena at Oxide Interfaces
}

\author{
H. Y. Hwang, ${ }^{1,2,3}$ Y. Iwasa, ${ }^{1,3,4}$ M. Kawasaki, ${ }^{1,3,4}$ B. Keimer, ${ }^{5}$ N. Nagaosa, ${ }^{1,4}$ Y. Tokura ${ }^{1,4,6}$ \\ (alphabetical order) \\ ${ }^{1}$ Correlated Electron Research Group (CERG) and Cross-Correlated Materials Research Group \\ (CMRG), RIKEN-Advanced Science Institute, Saitama 351-0198, Japan \\ ${ }^{2}$ Department of Applied Physics and Stanford Institute for Materials and Energy Science, \\ Stanford University, Stanford, CA 94305, USA \\ ${ }^{3}$ CREST, Japan Science and Technology Agency (JST), Tokyo 102-0075, Japan \\ ${ }^{4}$ Department of Applied Physics and Quantum-Phase Electronics Center (QPEC), University of \\ Tokyo, Bunkyo-ku, Tokyo 113-8656, Japan \\ ${ }^{5}$ Max Planck Institute for Solid State Research, 70569 Stuttgart, Germany \\ ${ }^{6}$ Multiferroics Project, ERATO, Japan Science and Technology Agency (JST), Tokyo 113-8656, \\ Japan
}

\section{BASIC Notions}

Transition metal oxides (TMOs) are an ideal arena for the study of electronic correlations because the $s$-electrons of the transition metal ions are removed and transferred to oxygen ions, and hence the strongly correlated $d$-electrons determine their physical properties such as electrical transport, magnetism, optical response, thermal conductivity, and superconductivity. These electron correlations prohibit the double occupancy of metal sites and induce a local entanglement of charge, spin, and orbital degrees of freedom. This gives rise to a variety of phenomena, e.g., Mott insulators, various charge/spin/orbital orderings, metal-insulator transitions, multiferroics, and superconductivity. ${ }^{1}$ In recent years, there has been a burst of activity to manipulate these phenomena, as well as create new ones, using oxide heterostructures. $^{2}$

Most fundamental to understanding the physical properties of TMOs is the concept of symmetry of the order parameter. As Landau recognized, the essence of phase transitions is the change of the symmetry. For example, ferromagnetic ordering breaks the rotational symmetry in spin space, i.e., the ordered phase has lower symmetry than the Hamiltonian of the system. There are three most important symmetries to be considered here. (i) Spatial inversion (I), defined as $r$ $\rightarrow$-r. In the case of an insulator, breaking this symmetry can lead to spontaneous electric 
polarization, i.e. ferroelectricity, or pyroelectricity once the point group belongs to polar group symmetry. (ii) Time-reversal symmetry ( $T$ ) defined as $t \rightarrow$-t. In quantum mechanics, the timeevolution of the wave-function $\psi$ is given by the phase factor $e^{-i E t / \hbar}$ with $E$ being the energy, and hence time-reversal basically corresponds to taking the complex conjugate of the wavefunction. Also the spin, which is induced by the "spinning" of the particle, is reversed by timereversal. Broken $T$-symmetry is most naturally associated with magnetism, since the spin operator changes sign with $T$-operation. (iii) Gauge symmetry $(G)$, which is associated with a change in the phase of the wave-function as $\psi \rightarrow e^{i \theta} \psi$. Gauge symmetry is connected to the law of charge conservation, and broken $G$-symmetry corresponds to superconductivity/superfluidity.

To summarize, the interplay among these electronic degrees of freedom produces various forms of symmetry breaking patterns of $I, T$, and $G$, leading to novel emergent phenomena, which can appear only by the collective behavior of electrons and cannot be expected from individual electrons. ${ }^{3}$ Figure 1 shows this schematically by means of several representative phenomena. From this viewpoint, the interfaces of TMOs offer a unique and important laboratory because $I$ is already broken by the structure itself, and the detailed form of broken $I$ symmetry can often be designed. Also, two-dimensionality usually enhances the effects of electron correlations by reducing their kinetic energy. These two features of oxide interfaces produce many novel effects and functions that cannot be attained in bulk form. Given that the electromagnetic responses are a major source of the physical properties of solids, and new gauge structures often appear in correlated electronic systems, we put "emergent electromagnetism" at the center of Fig. 1.

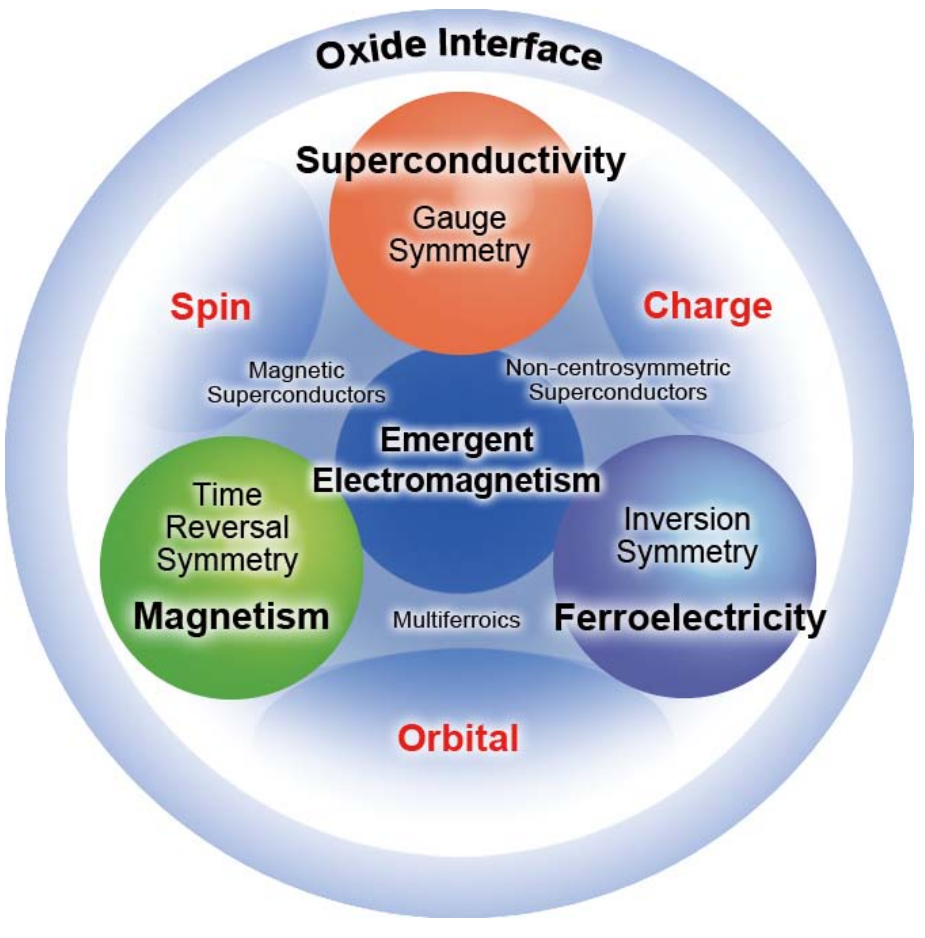


Fig. 1. Conceptual figure for the symmetries and degrees of freedom of correlated electrons which can be engineered at oxide interfaces.

Now we discuss some basic features of TMOs and their interfaces. Figure 2a shows the representative crystal structure of TMOs, i.e., perovskite. A transition metal ion (M) is surrounded by 6 oxygen $(\mathrm{O})$ atoms, which produce a crystal field acting on $\mathrm{M}$ with cubic symmetry. As a consequence, the originally 5-fold degenerate $3 d$ orbitals are split into 3-fold degenerate $t_{2 g}$ orbitals ( $x y, y z, z x$ orbitals), and 2-fold degenerate $e_{g}$ orbitals $\left(x^{2}-y^{2}, 3 z^{2}-r^{2}\right.$ orbitals). These orbitals have a different sign for the wave-function depending on the direction, which sometimes results in the cancellation of the overlap integrals with the p-orbitals of the oxygen atoms between two neighboring $M$ ions. Figure $2 b$ shows the three cases which survive this cancellation and lead to a nonzero amplitude for hopping between the two orbitals across an interface ( $x y$-plane). (Note that the hopping occurs due to second order processes by using two hops between the $d$-orbitals and the oxygen $p$-orbitals.) For all the other combinations of the orbitals, the hopping matrix element vanishes.

\section{a $\mathrm{AMO}_{3}$ (Perovskite)}
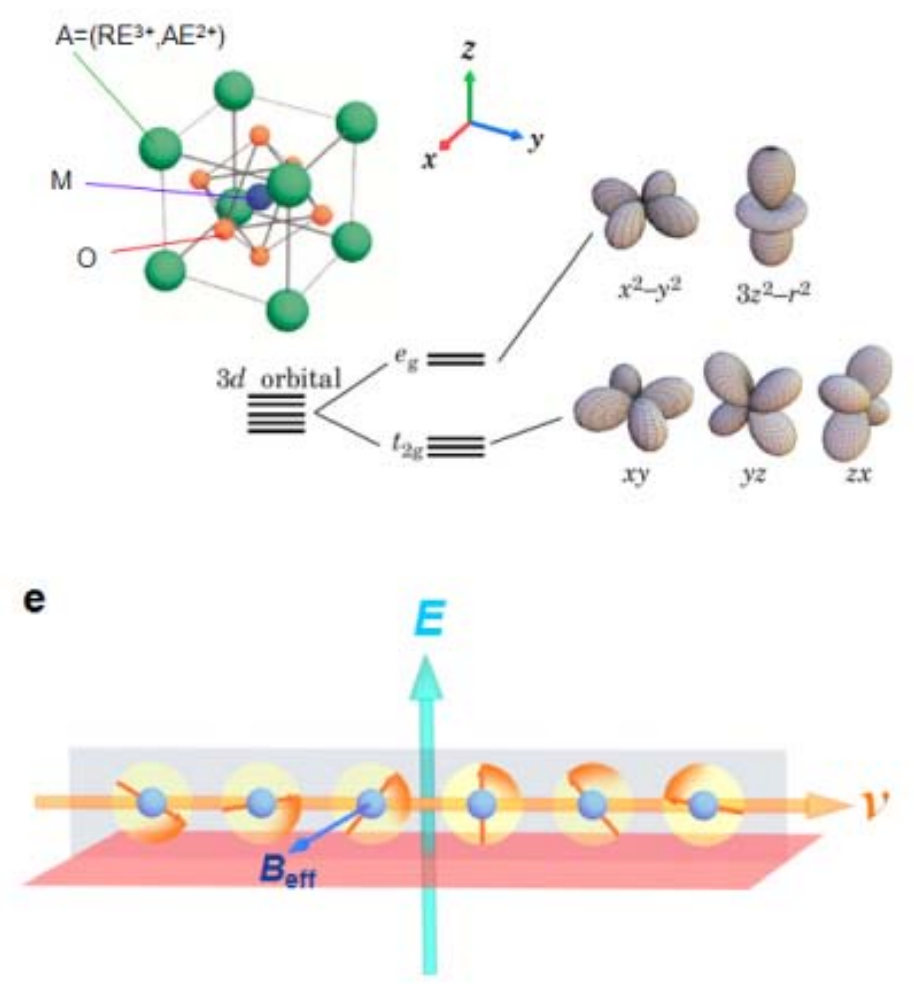

b
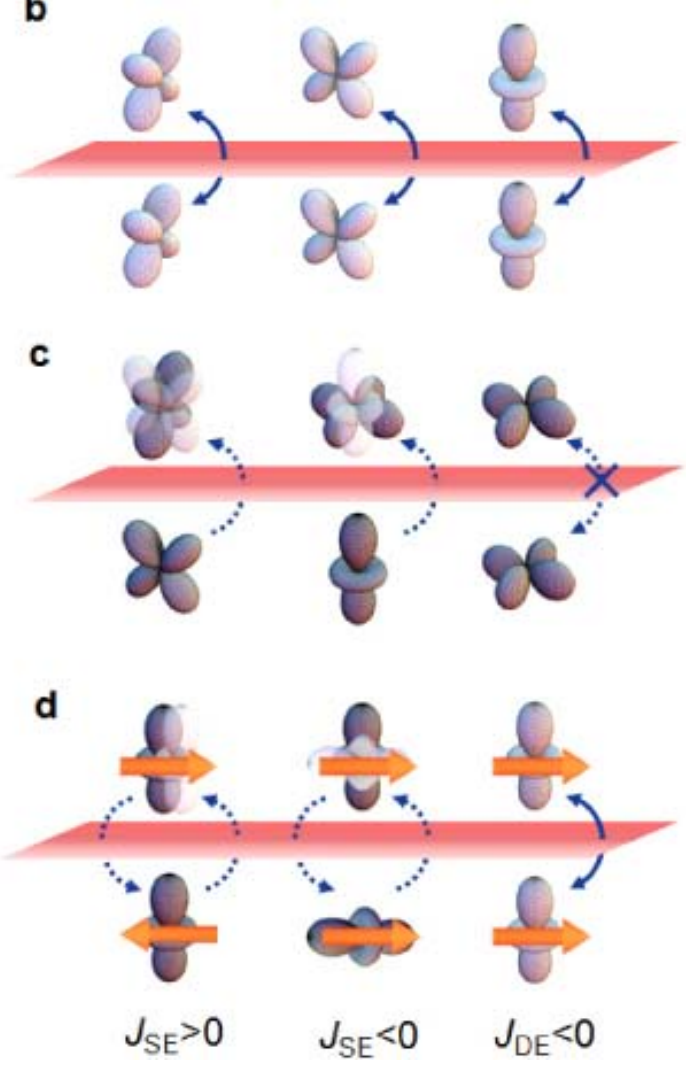
Fig. 2. a, Perovskite structure and orbital energy levels under the cubic crystal field due to oxygen atoms. b, Allowed hopping matrix elements between orbitals across the interface. c, Allowed virtual hopping matrix elements across the interface. The lightly colored orbitals are the destination of the virtual hopping process, while the electron is occupied in the darkly colored orbital. d, Various exchange interactions across the interface ( $J>0$, antiferromagnetic; $J<0$, ferromagnetic). Left: antiferromagnetic super-exchange interaction $J_{\mathrm{SE}}$ using the same orbital in the intermediate state. Middle: ferromagnetic super-exchange interaction between different orbitals. Right: double-exchange interaction $J_{\mathrm{DE}}$ where the real hopping of the conduction electron between the two $\mathrm{M}$ ions mediates the ferromagnetic coupling. e, Rashba-type spin-orbit interaction (SOI); the internal or applied electric field can produce an effective magnetic field $\left(B_{\text {eff }}\right)$ via the SOI to cause spin precession for the moving electron, or cycloidal spin modulation for localized spins.

Next we consider the correlation effect due to the Coulomb interaction on each $\mathrm{M}$ ion. By this interaction, spin and orbital moments are induced, and the exchange interactions between the two $\mathrm{M}$ ions across the interface are of fundamental importance. To consider the exchange processes, virtual hopping, i.e., the virtual transfer of the electron from the occupied orbital to the higher energy unoccupied orbital, is indispensable. Figure 2c illustrates two of the possible virtual hopping processes (left and middle), and a forbidden case (right). By using these virtual hopping processes twice, the super-exchange interactions are mediated, as shown in Fig. 2d. In the intermediate virtual states, the two electrons are occupying the same $M$ ion and hence the energy is increased by $U$ (on-site Coulomb repulsion). When the two orbitals are the same (left), Pauli's exclusion principle prohibits this second order process for parallel electron spins and hence the antiferromagnetic interaction results, while the ferromagnetic interaction results when the orbitals are different (middle). Figure 2d (right) illustrates the ferromagnetic double-exchange interaction mediated by conduction electrons hopping between the two $\mathrm{M}$ ions.

Up to now, we have not considered relativistic effects. Consider the electron moving with velocity $\vec{v}$ next to the interface, subject to the electric field $\vec{E}$ due to the broken $I$-symmetry (Fig. 2e). Then in the rest frame of the electron, there appears a magnetic field $\vec{B}_{\text {eff }}=\vec{v} \times \vec{E}$ according to the Lorentz transformation law, which is coupled to the magnetic moment of the electron spin $\vec{s}$ as described by the Hamiltonian $H_{S O} \propto \vec{B}_{\text {eff }} \cdot \vec{s}=(\vec{v} \times \vec{E}) \cdot \vec{s}$. This is exactly the relativistic spinorbit interaction (SOI). When $\vec{E} / / z$ and $\vec{v} / / y$ as in Fig. 2e, $\vec{B}_{e f f} / / x$ and spin precession occurs in the $y z$-plane. This represents the Rashba-type SOI. ${ }^{4}$

With these fundamentals of correlated electrons at interfaces, we will describe below how to design and realize various novel functions and phenomena such as charge/orbital/spin reconstructions, magneto-electric coupling, superconductivity, and the quantized Hall effect. 


\section{ChARgE/ORBITAL/SPIN RECONSTRUCTIONS}

Given that the physical properties of TMOs are often dominated by the state variables of the $d$-electrons, a natural starting point for the study of their heterostructures (Fig. 3a) is to determine their configuration at interfaces. Namely, what arrangements of the charge, spin, and orbital degrees of freedom can be induced, particularly those which have no bulk analog? While some aspects of recent findings have a direct correspondence in semiconductor or metal interfaces, manipulating interface states in TMOs can have striking consequences. It should be noted that the rapid growth of this field has been enabled by fabrication techniques with the prerequisite atomic-scale control, as this is often a very local phenomenon in oxides.

\section{Charge Transfer}

At any interface between electronic materials, charge can be transferred to equilibrate the electron chemical potential, leading to interface screening in metals, or band bending and depletion in semiconductors. This issue is already more complex for many TMOs, for which the "semiconductor" or "metal" is often derived by doping away from a Mott insulator. Since this state arises from strong electron interactions, the equivalent of a "band-bending" profile can have much more spatial structure than conventionally understood, reflecting features in the electronic

compressibility. ${ }^{5}$ Furthermore, the region over which the local carrier density varies can traverse entire phase diagrams, spanning metallic, magnetic, and superconducting phases. 

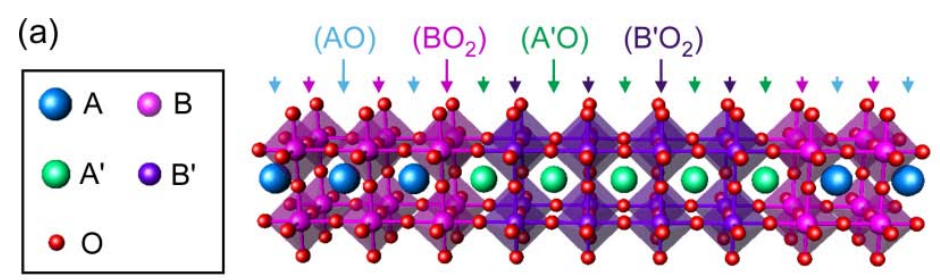

(b)

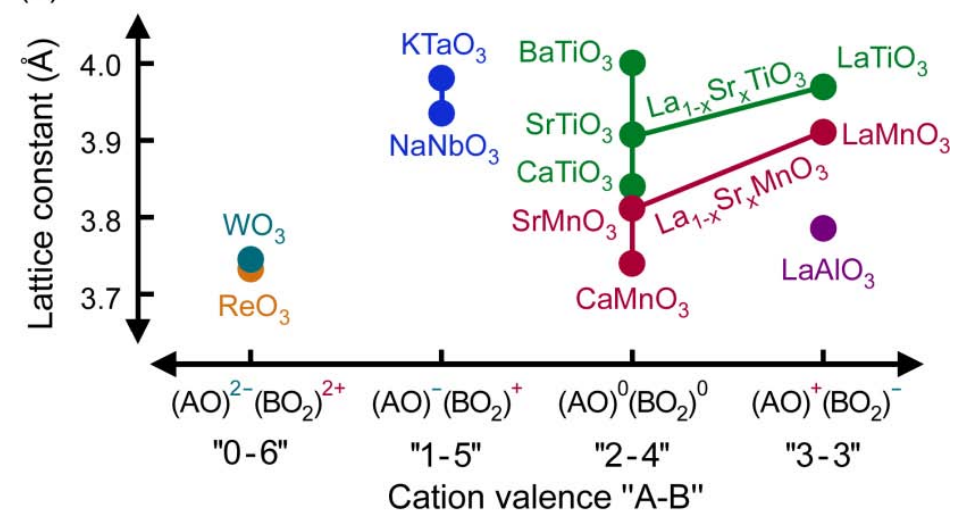

(c) $\quad \mathrm{LaTiO}_{3}$ in $\mathrm{SrTiO}_{3}(001)$

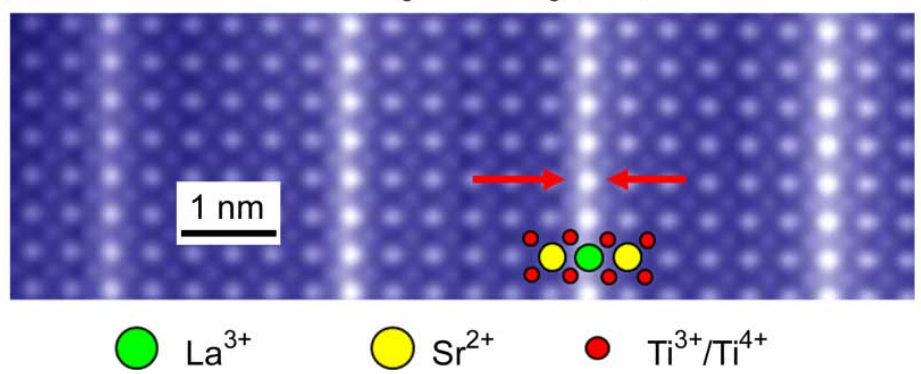

Fig. 3. a, Schematic illustration of ideal heterointerfaces between two perovskites, $\mathrm{ABO}_{3}$ and $\mathrm{A}^{\prime} \mathrm{B}^{\prime} \mathrm{O}_{3}$ stacked in the [001] direction. b, Representative lattice constants for various perovskites as a function of their charge sequence. c, Scanning transmission electron microscopy image of a $\mathrm{LaTiO}_{3} / \mathrm{SrTiO}_{3}$ superlattice. ${ }^{10}$

An early example of these issues arose in the study of the $\mathrm{CaMnO}_{3} / \mathrm{CaRuO}_{3}$ heterointerface between an antiferromagnetic insulator and a paramagnetic metal. ${ }^{6}$ Despite these constituents, the net result is a ferromagnetic interface, from which finite charge transfer between these layers can be deduced. In particular, the ferromagnetic magnetization was found to scale with the number of interfaces, rather than the bulk volume of the constituents. Even in the absence of real charge transfer, geometric control of virtual charge exchange can completely alter the magnetic structure of the interface, following the Kanamori-Goodenough rules for super-exchange coupling. ${ }^{7,8,9}$

Subsequently, a number of studies probed the electronic structure of interfaces between materials with different valence states of a given transition metal ion. Figure 3c shows an electron microscopy image of a superlattice composed of $\mathrm{SrTi}^{4+} \mathrm{O}_{3}$ and $\mathrm{LaTi}^{3+} \mathrm{O}_{3}$, varied down to the level of individual perovskite unit cells. ${ }^{10}$ Due to charge transfer, this heterostructure 
composed of band insulator and Mott insulator components nevertheless exhibited metallic behaviour. This can be understood as the nanoscale decomposition of the $(\mathrm{La}, \mathrm{Sr}) \mathrm{TiO}_{3}$ bulk solid solution, which has been discussed in terms of "doping" the empty $d$-band of $\mathrm{SrTiO}_{3}$ up to half filling. ${ }^{11}$ Rather than a strongly disordered average value, local variations in the valence state could be organized and studied for the first time. Furthermore, the region of this variation can be considered to cross the entire bulk phase diagram. ${ }^{11}$ However, this occurs in two-dimensions, not three, and among the underlying synthetic opportunities at interfaces is to change the balance of competing ground states, or enhance quantum fluctuations, and thereby create new states inaccessible in bulk materials.

Indeed, most of the notable bulk physical properties of TMOs arise from doping away from an integer valence state, and thus many studies are ongoing to examine interfaces between various constituents. For example, $\mathrm{LaMn}^{3+} \mathrm{O}_{3}$ and $\mathrm{SrMn}^{4+} \mathrm{O}_{3}$ are both antiferromagnetic insulators, but their superlattices exhibit transitions to the ferromagnetic metallic state and associated "colossal magnetoresistance". ${ }^{12,13,14}$ Another intriguing system is the interface between insulating $\mathrm{La}_{2} \mathrm{CuO}_{4}$ and metallic overdoped $(\mathrm{La}, \mathrm{Sr})_{2} \mathrm{CuO}_{4}$. These materials are on either side of the high temperature superconducting "dome" of the $T_{\mathrm{c}}$ vs. doping curve, but via charge transfer, superconductivity is found just at the interface. ${ }^{15,16}$ It has been suggested that such a separation of a region of strong pairing potential, and a region of high superfluid density, may be an avenue to enhancing $T_{\mathrm{c}}$ at interfaces. ${ }^{17,18}$

\section{Electrostatic Boundary Conditions in Heterostructures}

The charge structure of the interface between $\mathrm{LaTiO}_{3}$ and $\mathrm{SrTiO}_{3}$, or $\mathrm{LaMnO}_{3}$ and $\mathrm{SrMnO}_{3}$, actually has two contributions: charge transfer as previously discussed, and net excess charge due to the electrostatic boundary conditions imposed. The second aspect can be more clearly seen between two perovskites with both cation sites that are different, and has been most heavily investigated for the $\mathrm{LaAlO}_{3} / \mathrm{SrTiO}_{3}$ interface. ${ }^{19}$ Namely, two different interfaces can be formed in the [001] direction, $\mathrm{AlO}_{2}-\mathrm{LaO}-\mathrm{TiO}_{2}$ and $\mathrm{AlO}_{2}-\mathrm{SrO}-\mathrm{TiO}_{2}$, where the choice lies in the middle atomic layer. This simple degree of freedom has important consequences - the first interface is found to be conducting, while the second interface is insulating.

In terms of electrostatics, the two choices for the connection between these two insulators impose opposite boundary conditions. In simple ionic terms, $\mathrm{LaAlO}_{3}$ is composed of charged layers $\left(\mathrm{La}^{3+} \mathrm{O}^{2-}\right)^{+}$and $\left(\mathrm{Al}^{3+}\left(\mathrm{O}^{2-}\right)_{2}\right)^{-}$, while the corresponding layers in $\mathrm{SrTiO}_{3}$ are charge neutral. Therefore, terminating the $\mathrm{LaAlO}_{3}$ on an atomic plane at the interface presents the same "polar catastrophe" well-known in surface science to drive reconstructions of polar surfaces, and also arising in polar/non-polar semiconductor heterointerfaces. ${ }^{20,21}$ In order to avoid this diverging interface energy, $+/-1 / 2$ compensating charge is required, depending on the interface. The interesting feature of TMOs is their ability to assume different valence states, which can be used to "create" this compensating charge. For the $\mathrm{AlO}_{2}-\mathrm{LaO}-\mathrm{TiO}_{2}$ interface, $\mathrm{Ti}^{3+}$ is indeed observed, ${ }^{22}$ and this electronic reconstruction ${ }^{23}$ can stabilize the interface and source mobile 
electrons. For the $\mathrm{AlO}_{2}-\mathrm{SrO}-\mathrm{TiO}_{2}$ interface, the source of charge was found to be oxygen vacancies, and here a change in stoichiometry is used for compensation via an atomic reconstruction.

These simplistic ionic considerations have been shown to be robust to varying degrees of covalency, as well as the localized or itinerant nature of the interface charge. ${ }^{24}$ Furthermore, they are always present even in real systems with finite inter-diffusion. Indeed, it is suggested that minimizing the electrostatic energy can be a driving force for interface mixing, fundamentally limiting interface abruptness in some cases. ${ }^{22}$ However, it should be noted that there is active debate over alternative origins for the interface conductivity observed. Perhaps the leading issue is the formation of bulk-like oxygen vacancies in the $\mathrm{SrTiO}_{3}$ near the interface, which provides free carriers. ${ }^{25,26,27}$ There is indeed a very strong dependence of the interface carrier density on the oxygen partial pressure during growth, ${ }^{19,28}$ or post-annealing, and the degree of intrinsic versus extrinsic contributions to the free carriers is under active current study. Nevertheless, a clear constraint on all of the bound and free charges of the system is set by the electrostatic boundary conditions, and equilibrated with respect to the electrochemical potential.

(a)

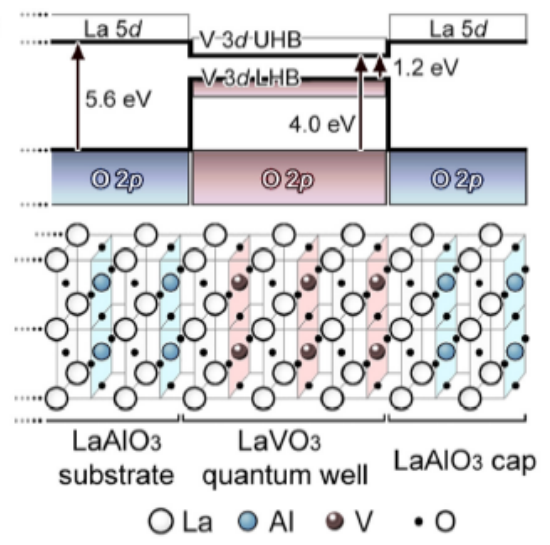

(b)

For a thick cap (decoupled) For a thinner cap

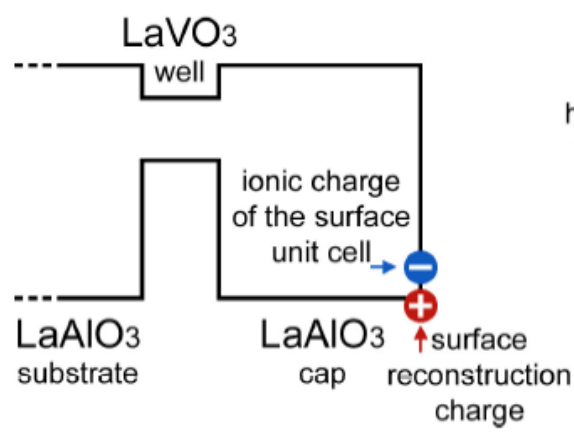

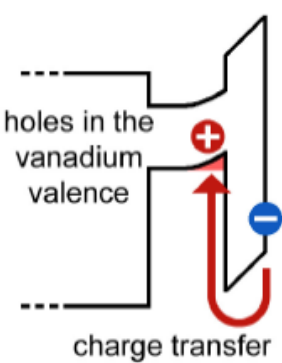

charge transfer

Fig. 4. a, Schematic band diagram and crystal structure of a $\mathrm{LaVO}_{3}$ quantum well embedded close to an $\mathrm{AlO}_{2}$-terminated $\mathrm{LaAlO}_{3}$ (001) surface. b, Illustrations showing how reconstruction charge at the $\mathrm{LaAlO}_{3}$ surface is transferred to the buried $\mathrm{LaVO}_{3}$ well. To resolve the "polar catastrophe" of the surface, positive charge is required. When the $\mathrm{LaAlO}_{3}$ cap is thick (left), the surface and the well are electrostatically decoupled, and the positive compensation charge is at the surface. For thinner spacing (right), the $\mathrm{LaVO}_{3}$ well accommodates this positive change, which is energetically more favored. ${ }^{32,34}$

One of the exciting nano-fabrication techniques that arise from these boundary conditions is the ability to write surface charge by atomic force microscopy, which has been demonstrated to 
induce conducting features on the few nanometer scale. ${ }^{29,30}$ This enables ready access to mesoscopic devices which are competitive with the most advanced e-beam lithography currently available. Another direction is to generalize these electrostatic constraints to other interface systems. ${ }^{31}$ As emphasized in Fig. 3, the "polarity mismatch" is quite general when considering oxide heterostructures with different components. A recent example is shown in Fig. 4, where a quantum well of the Mott insulator $\mathrm{LaVO}_{3}$ is placed in close proximity to the polar $\left(\mathrm{Al}^{3+}\left(\mathrm{O}^{2-}\right)_{2}\right)^{-}$ surface of $\mathrm{LaAlO}_{3}{ }^{32}$ While in isolation, this polar surface reconstructs via oxygen vacancies and lattice distortions, ${ }^{33}$ on short length scales it can alternatively modulation dope holes in the $\mathrm{LaVO}_{3}$ well. ${ }^{34}$ Thus there is a wide variety of doping and valence change schemes that are accessible in heterostructures that do not require local chemical doping, and are therefore free from the strong disorder typically introduced in bulk.

\section{Spin and Orbital Reconstructions}

In the previous sections, we have discussed the rearrangement of charge at oxide interfaces, which is driven primarily by electrostatic interactions. Because of the strong correlation between charge, spin, and orbital degrees of freedom, modulations of the charge density in TMOs can readily lead to spin or orbital polarization. One example we have already encountered is the $\mathrm{CaMnO}_{3}-\mathrm{CaRuO}_{3}$ interface, where transfer of a small density of itinerant charge carriers enhances the ferromagnetic double-exchange between $\mathrm{Mn}$ spins at the expense of antiferromagnetic super-exchange interactions, and hence induces ferromagnetic spin polarization at the interface., ${ }^{65}$ An example of charge-driven orbital polarization has been observed in the $\mathrm{LaAlO}_{3} / \mathrm{SrTiO}_{3}$ system, also discussed above, where the polarity mismatch (combined with the incipient ferroelectricity of $\mathrm{SrTiO}_{3}$ ) induces substantial lattice deformations, ${ }^{36}$ which in turn lead to a rearrangement of the level hierarchy of the partially occupied Ti $t_{2 g}$ orbitals through crystal-field effects. ${ }^{37}$

Another source of orbital and spin polarization in oxide heterostructures is epitaxial strain resulting from the mismatch of the lattice parameters of the TMO constituents. In analogy to the size mismatch between anions and cations in bulk TMOs, this strain is accommodated by a combination of uniform deformations and staggered rotations of the metal-oxide octahedra, which influence the orbital occupation via the crystal field. Lattice deformations resulting from epitaxial strain are ubiquitous in oxide heterostructures and superlattices. Ultrathin TMO films have served as model systems for studies of their influence on the competition between different spin-orbital ordering patterns in the plane of the film. ${ }^{38,39}$ However, unlike the charge-driven reconstructions discussed above which can be effectively screened at least in metallic or highly polarizable dielectric TMOs, the strain-driven spin and orbital polarization is typically maintained over a spatial range of tens of nanometers.

Right at the interface, the local crystalline environment of the transition metal ions differs strongly from the bulk-like environment just one unit cell away (Fig. 5). This implies large local differences in both the crystal field and in the network of covalent exchange bonds between 
metal-ion pairs via bridging oxygen ions. These differences can trigger genuine interfacial "spinorbital reconstructions" not secondary to charge rearrangements or epitaxial strain. This type of reconstruction has been predicted ${ }^{40,41,42,43,44}$ and observed ${ }^{45,46}$ at interfaces between TMOs with partially occupied, nearly degenerate $d$-orbitals, such as $\mathrm{LaTiO}_{3}$ or $\mathrm{LaNiO}_{3}$, with a chemically distinct wide-bandgap insulator such as $\mathrm{LaAlO}_{3}$ (Fig. 5). The loss of covalent metal-oxygenmetal bonds at the interface raises the energy of $d$-orbitals with lobes pointing towards the interface and leads to preferential occupation of orbitals polarized parallel to the interface.

The converse effect has been observed at interfaces of superconducting $\mathrm{YBa}_{2} \mathrm{Cu}_{3} \mathrm{O}_{7}{ }^{47,48}$ and multiferroic $\mathrm{BiFeO}_{3}{ }^{49}$ with the metallic ferromagnet $\mathrm{La}_{0.7} \mathrm{Ca}_{0.3} \mathrm{MnO}_{3}$, where new exchange bonds are formed across the interface and induce interfacial orbital and spin polarization. These latter systems are, however, also strongly affected by charge transfer across the interface, which has to be taken into account in order to arrive at a quantitative description of the experimental results. Other systems with strongly entangled charge, spin, and orbital degrees of freedom are interfaces of the orbitally nearly degenerate antiferromagnetic Mott insulators $\mathrm{LaTiO}_{3}$ and $\mathrm{LaVO}_{3}$ with $\mathrm{SrTiO}_{3}$, already encountered above, where the polarization mismatch between the constituents generates interfacial charge density profiles that cannot be obtained by chemical substitution in the bulk. For these systems novel interfacial charge, spin, and orbitally ordered states have been predicted. ${ }^{40,41,50}$ Direct observation of the corresponding ordering patterns lateral to the interface remains a challenge for experimental research.

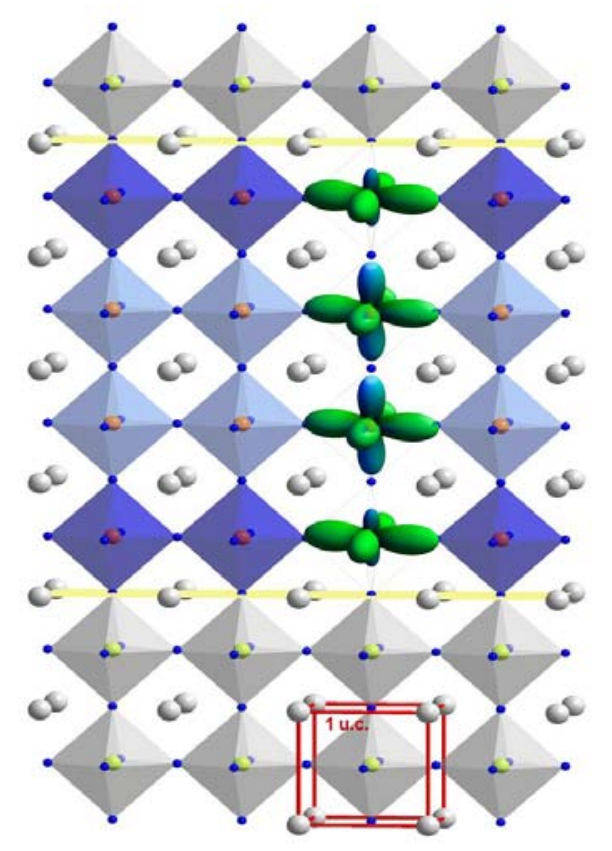

Fig. 5. Heterostructure of metallic $\mathrm{LaNiO}_{3}$ with partially occupied $\mathrm{Ni} e_{g}$ orbitals (blue) and insulating $\mathrm{LaAlO}_{3}$ (white). ${ }^{46}$ The orbital polarization at the interface is exaggerated for clarity. 


\section{EMERGENT INTERFACE STATES}

As was discussed in the previous section, interfaces of TMOs exhibit various interesting properties, ranging from two-dimensional confinement of electronic states that can be naively expected from carrier-doped bulk solid solution analogs, to emergent phenomena that are hard to predict due to strong correlation effects of $d$-electrons as well as reconstructions of their various degrees of freedom. Referring to the discussion shown in Fig. 1, the concomitant presence of two broken symmetries is one of the most intriguing subjects of near-future research of TMO interfaces. Given that any single interface structurally breaks I-symmetry, inducing magnetism or superconductivity is an approach to examine the emergent properties and functionalities of TMO interfaces.

\section{Magneto-Electric Coupling in Tricolor Superlattices}

Both $\mathrm{CaMnO}_{3} / \mathrm{CaRuO}_{3}$ and $\mathrm{LaMnO}_{3} / \mathrm{SrMnO}_{3}$ interfaces are known to show ferromagnetism due to the effective doping induced by charge transfer. ${ }^{6,12,13,14}$ However, the magnetization $(\vec{M})$ induced at a single interface is often too small to be detected by conventional tools and it is hard to characterize the cross-coupling of $\vec{M}$ with electric polarization $(\vec{P})$, such as magneto-electric effects. One of the neat ways to amplify the effect is making superlattices to multiply the signal. In conventional $\mathrm{ABAB}$...superlattices, however, $\vec{P}$ has the opposite sign between $\mathrm{AB}$ and $\mathrm{BA}$ interfaces which largely cancel each other. In tricolor ABCABC...superlattices, all the $\vec{P}$ at the $\mathrm{AB}(\mathrm{BC})$ interfaces can be arranged to have the same sign. In such a case the toroidal moment ( $\vec{T}$ ) is defined as $\vec{T}=\frac{1}{2} \sum_{i} \vec{r}_{i} \times \vec{S}_{i}$ (here $\vec{r}_{\mathrm{i}}$ and $\vec{S}_{\mathrm{i}}$ are the electron coordinates and spin components at the $i^{\text {th }}$ atomic site), which remains finite. A non-vanishing toroidal moment is equivalent to a built-in vector potential under the presence of spin-orbit coupling, thereby producing magnetoelectric action.

Accumulating $\vec{P}$ at each interface was predicted and demonstrated in $\mathrm{CaTiO} / 3 / \mathrm{SrTiO}_{3} / \mathrm{BaTiO}_{3}$ ferroelectric-dielectric superlattices to show spontaneous $\vec{P}$ across the entire superlattice. ${ }^{51,52,53}$ By combining ferromagnetic ( $\mathrm{La}, \mathrm{Sr}) \mathrm{MnO}_{3}$ with two different dielectrics, $\mathrm{La}^{3+} \mathrm{Al}^{3+} \mathrm{O}_{3}$ and $\mathrm{Sr}^{2+} \mathrm{Ti}^{4+} \mathrm{O}_{3}$, an artificial polar ferromagnet could be constructed. ${ }^{54,55} \mathrm{~A}$ similar or even more straightforward example is isolated interfaces combining $\mathrm{LaMnO}_{3}$ and $\mathrm{SrMnO}_{3}$, which are separated by a third intervening layer of magnetically inactive $\mathrm{LaAlO}_{3}$ (Fig. 6a); $\mathrm{LaMnO}_{3}$ and $\mathrm{SrMnO}_{3}$ are originally antiferromagnets, layered-type and G-type respectively, but their interface becomes ferromagnetic, and importantly polar as well due to interface charge-transfer and the resultant double-exchange interaction as discussed above. ${ }^{56}$ Here, the concomitant presence of $\vec{P}$ and $\vec{T}$ in these tricolor superlattices is verified using second harmonic (SH) generation and its magneto-optical rotation, respectively. The former is rather straightforward; incident laser light with electric field parallel to the surface (s-polarization) generates frequency-doubled light with 
$p$-polarization, through the standard nonlinear effect due to $\vec{P}$ perpendicular to the surface. Since $\vec{T}$ is orthogonal to both $\vec{M}$ and $\vec{P}$, by inverting the direction of $\vec{M}$ by applied magnetic field, $\vec{T}$ changes its sign ( $\vec{P}$ is invariant, being structurally defined). This in-plane $\vec{T}$ generates a component of s-polarized $\mathrm{SH}$ response. Combined with the p-polarized $\mathrm{SH}$ component, this results in Kerr rotation of the SH light, as exemplified in Fig. 6 b.

It is noteworthy in Fig. 6c that the magnitude of $\vec{M}$ as well as the the Kerr rotation angle $\Phi$ depends critically on the epitaxial strain. This is perhaps related with the effect of orbital reconstruction; the orbital occupation at the ferromagnetic interface prefers the orbital state $x^{2}-y^{2}$ in the case of a tensile-strained interface $\left(\mathrm{SrTiO}_{3}\right.$ substrate in Fig. 6c), while the orbital states $x^{2}$ $y^{2}$ and $3 z^{2}-r^{2}$ are degenerate in the cubic case $\left(\mathrm{La}_{0.3} \mathrm{Sr}_{0.7} \mathrm{Al}_{0.65} \mathrm{Ta}_{0.35} \mathrm{O}_{3}\right.$ substrate). The latter gives larger $\vec{M}$ (and possibly larger $\vec{P}$ ) due to enhanced charge transfer as shown in Fig. 2, resulting in larger $\vec{T}$ and hence $\Phi$. One of the applications of this technique is to selectively detect ferromagnetic order near the interface. In magnetic tunnel junctions, for example, the important parameter for tunneling magneto-resistance (TMR) is the spin polarization of charge carriers at the interface. In the case of TMOs, the competition between ferromagnetic and antiferromagnetic order tends to cause spin canting at the interface, which reduces the spin polarization and deteriorates the TMR. This SH technique could detect such a subtle variation of the interface $\vec{M}$ with the change of magnetic field for the $(\mathrm{LaSr}) \mathrm{MnO}_{3} / \mathrm{SrTiO}_{3}$ interface, proving that the overdoping of holes due to charge transfer biased the ferromagnetic interface towards antiferromagnetism or caused spin canting. ${ }^{57}$
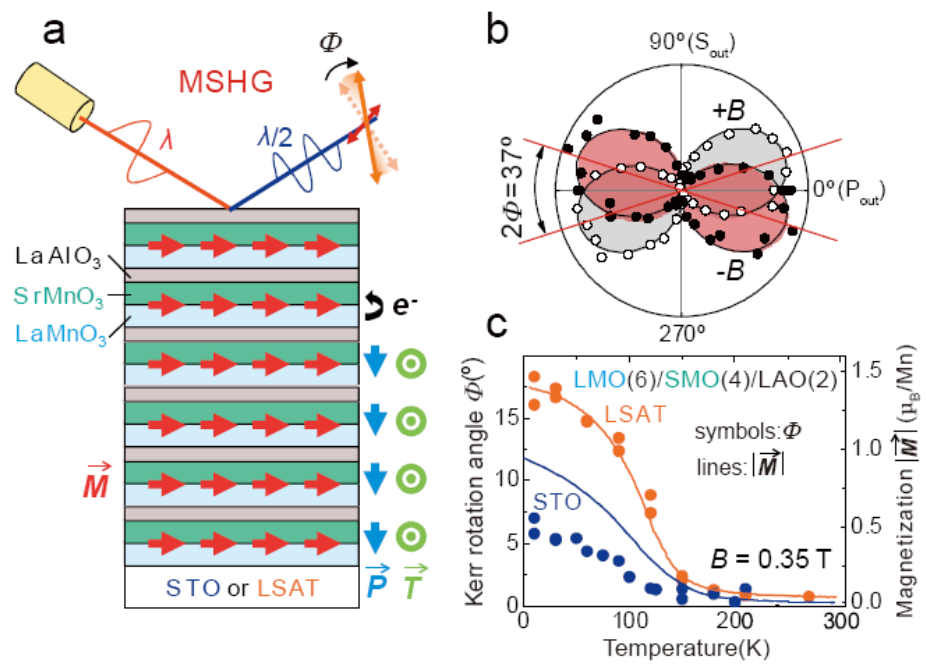

Fig. 6. a. A schematic of magnetization induced second harmonic generation (MSHG) for a tricolor superlattice composed of $\mathrm{LaMnO}_{3}$ (LMO), $\mathrm{SrMnO}_{3}$ (SMO), and $\mathrm{LaAlO}_{3}$ (LAO). For controlling the in-plain lattice strain and resulting preference in orbital occupancy, $\mathrm{La}_{0.3} \mathrm{Sr}_{0.7} \mathrm{Al}_{0.65} \mathrm{Ta}_{0.35} \mathrm{O}_{3}$ (LSAT; nearly lattice matched) and $\mathrm{SrTiO}_{3}$ (STO; tensile-strained) substrates were employed. Using incident s-polarized fundamental light $(\lambda=800 \mathrm{~nm})$, the 
polarization characteristics are analyzed for SH light $(\lambda / 2)$. b. Analyzer angle dependence of the SH intensity. c, Kerr rotation angle (circles) and magnetization (lines) of superlattices with the same structure (6 unit cells (uc) LMO, 4 uc SMO, and 2 uc LAO) on different substrates. (Data courtesy of Th. Lottermoser and H. Yamada.)

\section{Interface Superconductivity}

(a)

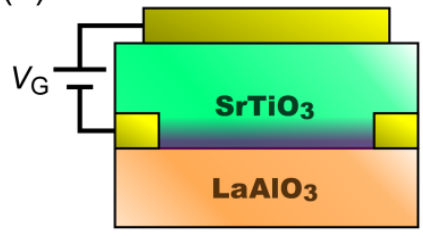

(c)

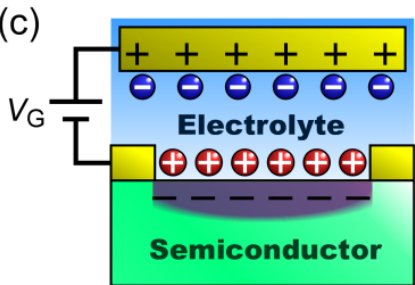

(b)

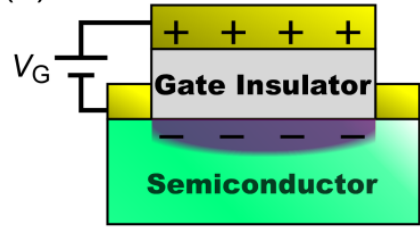

(d)

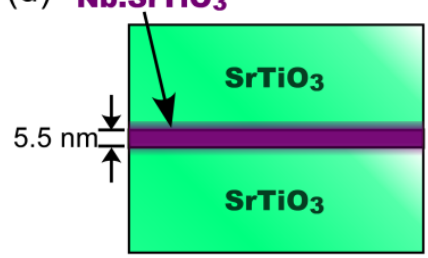

(e)

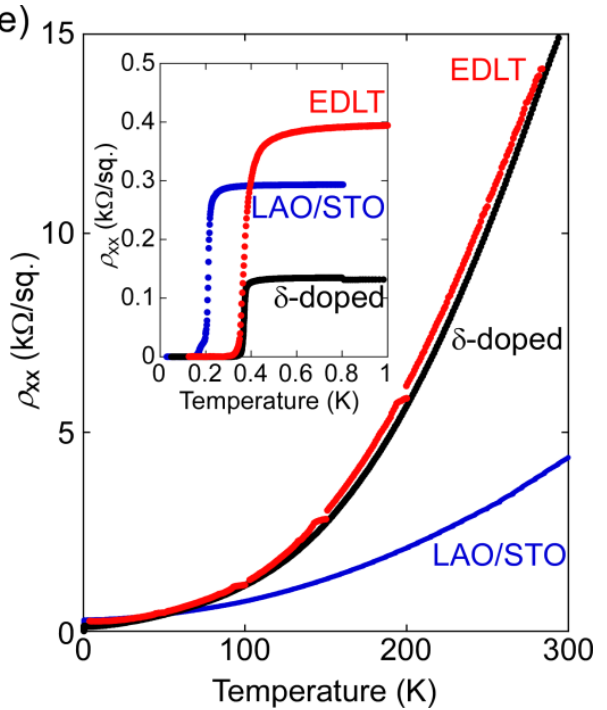

Fig. 7. Various device structures used to produce 2D/interface superconductivity. a, $\mathrm{LaAlO}_{3} / \mathrm{SrTiO}_{3}$ heterostructures with a gate electrode attached on the substrate side of $\mathrm{SrTiO}_{3} . \mathbf{b}$, MOS-FET type device. c, EDLT configuration. d, $\delta$-doped quantum well using $\mathrm{SrTiO}_{3}$. The 2DEG is indicated in purple. e, Comparison of the temperature dependence of the sheet resistance and interface superconductivity of $\mathrm{SrTiO}_{3}$ emerging at the $\mathrm{LaAlO}_{3} / \mathrm{SrTiO}_{3}$ interface (blue, after Ref. 60), EDLT at $V_{\mathrm{G}}=3.5 \mathrm{~V}$ (red, after Ref. 63), and $\delta$-doped quantum well (black, after Ref. 70). The inset is a blowup of the low temperature region.

Creation and control of interface superconductivity in TMOs are attracting growing interest over the last two decades. The most well-known example is the $\mathrm{LaAlO}_{3} / \mathrm{SrTiO}_{3}$ heterostructure shown in Fig. 7a, which is an archetypal polar catastrophe interface discussed previously. Although the two constituent materials are both wide-gap insulators, a two-dimensional electron gas (2DEG) is generated at their interface, having high enough mobility to display quantum oscillations in magnetotransport. ${ }^{19}$ Furthermore, this 2DEG exhibits $2 \mathrm{D}$ superconductivity at $T_{\mathrm{C}}=$ $0.2 \mathrm{~K}^{58}$ This unexpected interface superconductivity is indicative of a further rich variety of emergent properties in TMO interfaces. More importantly, due to the large dielectric constant of the $\mathrm{SrTiO}_{3}$ substrate, the 2DEG can be modulated by the back-gate configuration as schematized in Fig. $7 \mathrm{a} .{ }^{59,60}$ The dome-shaped superconducting phase diagram in the $T_{\mathrm{C}}-V_{\mathrm{G}}$ plane obtained 
on a single device is reminiscent of the $\mathrm{SrTiO}_{3}$ bulk phase diagram, ${ }^{61}$ demonstrating high tunability of the TMO interfaces.

More versatile gate-tunability is seen in the conventional metal-oxide-semiconductor field effect transistor (MOS-FET) configuration (Fig. 7b). The advantage of electrostatic carrier doping in a MOS-FET includes not only high tunability but also absence of chemical disorder, in contrast to chemical doping. The MOS-FET structures have been indeed successful in electric field-control of superconductivity in cuprate ultra-thin films. ${ }^{62}$ However, superconductivity can be gate-controlled only when it is assisted by chemical doping, because the electrostatically accumulated charge density in MOS-FETs is not sufficient for inducing superconductivity in undoped insulators.

Recently, a different type of field effect device, the electric double layer transistor (EDLT), was found to have the ability to induce superconductivity in insulators free from chemical doping. ${ }^{63}$ A device configuration is illustrated in Fig. 7c. Instead of solid gate dielectrics, the EDLT employs ionic conductors like electrolytes or ionic liquids. At the semiconductor/electrolyte interface, an electric double layer (EDL), a self-organized capacitor with sub-nanoscale thicknesses, is formed. ${ }^{64}$ The working mechanism of EDLTs resembles that of solid gated MOS-FETs. The most crucial difference is that the gate voltage $V_{\mathrm{G}}$ predominantly drops at the EDL interface, so that the electric field produced is on the order of $10 \mathrm{MV} / \mathrm{cm}$, allowing high density charge accumulation reaching $10^{15} \mathrm{~cm}^{-2}{ }^{65}$ This value is $1-2$ orders of magnitude larger than that achieved in solid gated MOS-FETs, which are breakdown limited. The application of $V_{\mathrm{G}}$ induced insulator-to-metal transitions followed by superconductivity, as first demonstrated in $\mathrm{SrTiO}_{3}\left(T_{\mathrm{C}}=0.45 \mathrm{~K}\right),{ }^{63}$ and then in $\mathrm{ZrNCl}\left(T_{\mathrm{C}}=15.2 \mathrm{~K}\right) .{ }^{66}$ The concept of the EDLT is quite versatile, and in fact, gate tuning of high $T_{\mathrm{c}}$ cuprates has been already tested. ${ }^{67}$ Furthermore, the EDLT technique can be used as a tool for searching for new superconductors.

So far, we have focused on single heterointerfaces including TMOs. However, it should be pointed out that a variety of artificially designed structures are nowadays available in the TMO superstructure systems. An intriguing structure is the $\delta$-doped quantum well of $\mathrm{SrTiO}_{3},{ }^{68,69}$ which also displays 2D superconductivity. ${ }^{70}$ Here a narrow region of single crystal $\mathrm{SrTiO}_{3}$ is chemically doped to spatially define a superconducting region. When the width of this region is reduced below the superconducting coherence length, there is a $3 \mathrm{D}-2 \mathrm{D}$ crossover in the superconducting state; similarly, on the de Broglie length-scale, there is a separate dimensional crossover for the normal state electrons, which exhibit sub-band quantization for narrow channels. ${ }^{71}$ Together with the flexibility to vary the local doping level, a rich phase diagram can be engineered to examine superconductor/normal-metal/insulator phase transitions in various dimensions. A notable feature of $\delta$-doping is that the mobility strongly increases in the 2D limit, in analogy to $\delta$-doping in semiconductors. This aspect suggests that a new regime of 2D superconducting phase transitions can be experimentally accessed approaching the clean limit. Furthermore, bilayer and superlattice structures can be designed to tune interlayer coupling in an 
artificial analogue of bulk layered superconductors, providing opportunities to address general questions of the role of interlayer coupling in quasi-2D superconductivity.

In addition to controllable carrier density, the interfaces like $\mathrm{LaAlO}_{3} / \mathrm{SrTiO}_{3}$ and $\mathrm{FET}$ devices offer a novel platform for Rashba physics, in which the spin-orbit interaction can be manipulated by the gate electric field. ${ }^{72,73}$ At any interface, inversion symmetry is inherently broken, but the gate effects allow us to control the strength of asymmetric potentials, which can be a great advantage for manipulation of spin polarization in TMO interfaces. In this sense, the interface superconductivity in $\mathrm{LaAlO}_{3} / \mathrm{SrTiO}_{3}$ as well as in MOS-FETs and EDLTs should be further considered as noncentrosymmetric systems. By contrast, the (un-gated) $\delta$-doped structure is inversion symmetric, and thus Rashba effects are excluded.

This contrast is perhaps highly relevant for the recent observations of the microscopic coexistence of ferromagnetism with superconductivity in $\mathrm{LaAlO}_{3} / \mathrm{SrTiO}_{3},{ }^{74,75,76}$ which is not found in $\delta$-doped $\mathrm{SrTiO}_{3}$. The fact that these normally antagonistic order parameters can be simultaneously observed indicates that this interface breaks all of the principal symmetries, $I, T$, and $G$, and with charge, ${ }^{22}$ orbital, $^{37}$ and spin reconstructions ${ }^{28}$ all at play. It is therefore an intriguing question whether this interface, composed of "standard" materials, can nevertheless exhibit exotic superconducting ${ }^{77}$ and edge state phenomena as a result of the formation of an emergent interface state.

\section{Fractional Quantum Hall Effect in $\mathrm{ZnO} /(\mathrm{MgZn}) \mathrm{O}$}

Charge accumulation at oxide interfaces has been used as a nice tool for realizing emergent phenomena as discussed above. When one employs the clean semiconductor ZnO, its 2DEG can exhibit the fractional quantum Hall effect, which is known to emerge only in extremely clean systems such as GaAs. ${ }^{78}$ The means of charge accumulation is, in some sense, similar to the polar catastrophe in the $\mathrm{LaAlO}_{3} / \mathrm{SrTiO}_{3}$ system. ${ }^{79} \mathrm{In} \mathrm{Mg}_{x} \mathrm{Zn}_{1-x} \mathrm{O} / \mathrm{ZnO}$ heterostructures, the mismatch in spontaneous polarization of the two piezoelectric compounds is compensated by the charge accumulation at the interface. ${ }^{80}$ Electrons are confined in the narrower band-gap material $\mathrm{ZnO}$ as shown in inset of Fig. 8a. The first demonstration of the quantum Hall effect in oxides was made possible in a $\mathrm{Mg}_{0.15} \mathrm{Zn}_{0.85} \mathrm{O} / \mathrm{ZnO}$ heterostructure fabricated by pulsed laser deposition, which achieved a maximum electron mobility $(\mu)$ of $5,500 \mathrm{~cm}^{2} \mathrm{~V}^{-1} \mathrm{~s}^{-1}$ (Ref. 80). The use of molecular beam epitaxy has since enabled a great reduction in the impurity concentration of the samples, resulting in a significant $\mu$ enhancement. ${ }^{81,82}$ Figure $8 \mathrm{a}$ shows an example of the magneto-transport properties for a $\mathrm{Mg}_{0.01} \mathrm{Zn}_{0.99} \mathrm{O} / \mathrm{ZnO}$ heterostructure with a charge carrier density $n=2 \times 10^{11} \mathrm{~cm}^{-2}$ and $\mu=300,000 \mathrm{~cm}^{2} \mathrm{~V}^{-1} \mathrm{~s}^{-1}$ which was top-gated. ${ }^{83}$ In addition to the integer states $(v=1,2, \ldots)$, many fractional states including the most fundamental $v=1 / 3$ are clearly seen.

Given increasingly higher mobility electrons at oxide interfaces, mesoscopic physics will become a new frontier, where electron coherence lengths exceed device size. For comparison 
with other semiconductors, we plot in Fig. 8b the transport scattering time $\tau_{\mathrm{tr}}=\mu \mathrm{m}^{*} / e$ to represent the cleanness of the systems hosting 2DEGs, where $e$ and $m^{*}$ are the elementary charge and the electron effective mass, respectively. Given its large $m^{*}$ and low dielectric constant $\varepsilon$, ZnO displays strong electron correlation effects as characterized by the Wigner-Seitz radius $r_{\mathrm{s}}$ $=1 / \sqrt{\pi n} a_{B}^{*}$, where $a_{B}^{*}$ is the effective Bohr radius $\left[\left(\varepsilon / \varepsilon_{0}\right) /\left(m^{*} / m_{0}\right)\right] a_{B}$ ( $\varepsilon_{0}$ is the vacuum permittivity, $m_{0}$ is the bare electron mass, and $a_{\mathrm{B}}$ is the Bohr radius). $r_{\mathrm{s}}$ values in current $\mathrm{ZnO}$ heterostructures have exceeded 10, which is difficult to achieve in other semiconductors while keeping a large $\tau_{\mathrm{tr}}$. By improving the growth conditions of $\mathrm{ZnO}$ heterostructures and their design, it is possible to reduce the charge carrier density, further increasing correlation effects, resulting in enhancements of the electron mass and spin susceptibility. A metal-insulator transition is expected in the low $n$ limit; theoretically Wigner crystallization is predicted to occur at $r_{\mathrm{s}}$ over 35 in $2 \mathrm{D} ;{ }^{84}$ in such a situation, new physics may be observed. Incorporating the spin degree of freedom also offers intriguing studies. In fact, the magnetic susceptibility is quite large for 2DEGs in $\mathrm{ZnO}$; the effective $g$ factor $\left(g^{*}\right)$ value is as large as 4 in contrast to about 0.5 in GaAs. ${ }^{85}$ Therefore, a quantum Hall ferromagnetic state can be formed. ${ }^{86}$ In addition, a $\mathrm{ZnO}$ heterostructure is almost free from nuclear spin - the natural abundance of $\mathrm{Zn}$ isotopes with nonzero spin $\left({ }^{67} \mathrm{Zn}\right)$ is $4 \%$, and that of oxygen with non-zero spin $\left({ }^{17} \mathrm{O}\right)$ is $0.038 \%$, and hence the electron - nuclear spin interaction is almost negligible. Therefore, $\mathrm{ZnO}$ is an ideal medium for spin transport experiments. In the same spirit, the spin of a localized electron, for instance in a quantum dot, can be used for storage of quantum information. Finally, $\mathrm{ZnO}$ heterostructures can be combined with a variety of material choices in oxides, with the aim of injecting spin-polarized carriers or superconducting Cooper pairs.
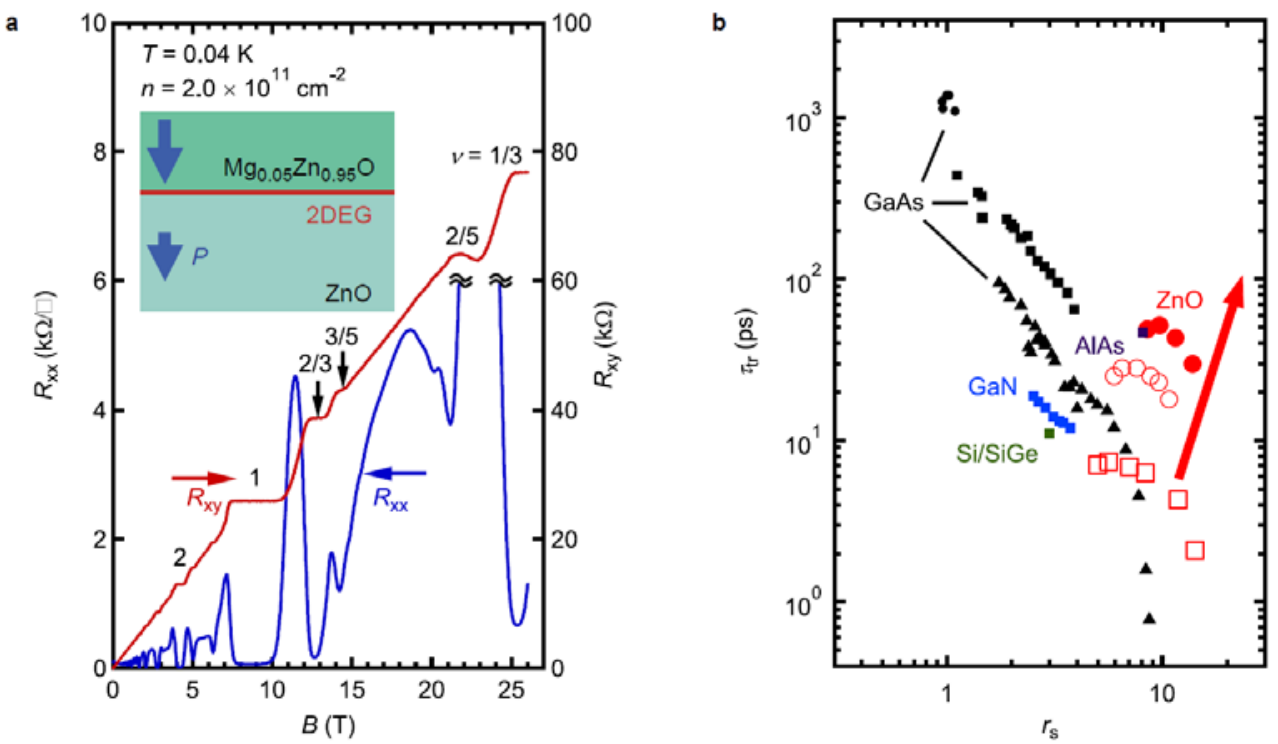
Fig. 8. a, Longitudinal resistance $R_{\mathrm{xx}}$ (blue) and Hall resistance $R_{\mathrm{xy}}$ (red) of a two-dimensional electron gas formed at a $\mathrm{Mg}_{0.01} \mathrm{Zn}_{0.99} \mathrm{O} / \mathrm{ZnO}$ interface. The inset depicts a cross-sectional schematic of the heterostructure. b, Comparison of 2DEGs in various semiconductors as functions of the electron-electron interaction strength represented by the Wigner-Seitz radius $r_{\mathrm{s}}$ and transport scattering time $\tau_{\text {tr }}$. Data are derived from Fig. 2 of Ref. 81 except for the solid red circles, obtained for the sample shown in a. The arrow indicates the direction of the progress in $\mathrm{ZnO}$ to pursue a regime of parameters that is hard to access in other semiconductors.

\section{OUTLOOK}

“The interface is the device,” stated Herbert Kroemer in his Nobel address. ${ }^{87}$ While this was in reference to semiconductors, it is particularly important for correlated oxides as well - namely, controlling the interface in terms of atomic, magnetic, orbital, and graded-potential design. As described in the preceding sections, TMOs host versatile forms of order in their charge, spin, and orbital sectors. These electronic orders meet together at the interface and are strongly affected by electronic processes such as charge-transfer, hybridization, and spin/orbital exchange interactions, as well as by the built-in scalar/vector potential, to produce emergent properties and functions. Here, let us briefly discuss possible outcomes of the on-going or near-future research on oxide interfaces in light of (1) Mottronics, (2) orbitronics, (3) spintronics, and (4) relativisticelectronics.

The term Mottronics (1) was coined to represent electronic functions emerging from the Mott transition, i.e. the metal-insulator transition in correlated-electron systems. The interface insulating state can be altered by charge transfer or a gate field to produce a conducting state via the Mott transition. The recently developed ionic-liquid gate method (EDLT) as described above can change the carrier concentration (or band filling) to a larger extent beyond the conventional dielectric solid-state gate and will enlarge the scope for electric-field induced phase changes at correlated oxide interfaces. An important outcome of this Mottronics is the resistive switching memory function, which can arise from an electric-field pulse induced phase change at an interface with a metal electrode. This function may be incorporated in resistance random-accessmemory (ReRAM), which is now under development towards industrial applications. ${ }^{88}$ One plausible mechanism of the nonvolatile resistance switching is the change of the Schottky barrier at the oxide/electrode interface, affected by the current pulse assisted movement of oxygens; ${ }^{89}$ note here that oxygen stoichiometry is directly related to the $d$-electron band filling and hence to the metal-insulator phenomena. Another interesting application is the photovoltaic effect at a Mott insulator junction ${ }^{90}$ or correlated solar cell. The gradient of potential can be built in for the Mott insulator junction as well as for the conventional semiconductor p-n junction to work as a separator of photogenerated electrons and holes. Multiple carrier generation in the single photon absorption process may be anticipated in Mott insulator junctions, as already demonstrated for the case of bulk Mott insulators or charge-ordered insulators. ${ }^{91}$ 
The control of the orbital degree of freedom of $d$-electrons is another important subject, and this would lead to (2) orbitronics function. With the change of the orbital state, magnetic, transport, and optical properties are significantly altered. In general, interface modification of the magnetic state, e.g. in the case of $\mathrm{LaMnO}_{3} / \mathrm{SrMnO}_{3}$, is inseparable from the orbital state modification. However, even if both phases on either side of the interface are ferromagnetic or metallic, their respective orbital polarizations will strictly gate $d$-electron hopping across the interface, as depicted in Figs. $2 \mathrm{~b}$ and c. Electric-field control or vertical-current switching of the orbital state at the interface will be a good target for the forthcoming orbitronics research. Engineering of spin-orbital Hamiltonians at interfaces may also lead to new routes for hightemperature superconductivity. ${ }^{42,43}$

Transition-metal oxides often host $d$-electron magnetism, and hence its interface modulation may lead to (3) spintonics functions. Spin-dependent tunneling through a barrier layer has been extensively studied in the context of the oxide TMR device, yet researchers have always encountered large modifications of the spin state at the interface even for an originally simple (half-metallic) ferromagnetic state; this easily degrades the TMR characteristics, in particular at elevated temperatures. Conversely, taking advantage of the spin sensitivity to the interface potential, the production of the ferromagnetic state at an oxide interface, say by electric field gating, will be a challenging subject in the context of spintronics applications. The ferromagnetic moment lying at an interface with broken inversion-symmetry gives rise to the toroidal moment (built-in vector potential), as discussed above. Gate electric-field control of the toroidal moment, if realized, would produce spectacular magneto-electric functions.

The last, but not least, promising direction is (4) relativistic-electronics, which employs the relativistic spin-orbit interaction (SOI). While this is often an integral component of spintronics in semiconductors and metals, it is only recently being fully appreciated for TMOs, and deserves separate mention. For example in $\mathrm{Sr}_{2} \mathrm{IrO}_{4}$, spin orbit splitting is comparable to $U$, and the insulating state is best described in terms of total angular momentum states. ${ }^{92}$ A representative heterostructure effect is the magneto-electric coupling in the spin transistor proposed by DattaDas $^{93}$ where the spin precession is manipulated by the electric field, as well as the spin-galvanic effect, i.e., the current-induced spin polarization or the current-control of the magnetization. ${ }^{94,95}$ When the time-dependence of the SOI is considered, more rich phenomena can be designed, including adiabatic spin pumping and the manipulation of quantum qubits by electric field. Even without mobile carriers, the coupling between the spin and electric field is induced by the SOI. For example, spin helicity can be controlled by electric field; through the SOI two non-collinear spins $\vec{S}_{i}$ and $\vec{S}_{j}$ produce the polarization $\vec{P} \propto \hat{e}_{i j} \times\left(\vec{S}_{i} \times \vec{S}_{j}\right)$ where $\hat{e}_{i j}$ is the unit vector connecting the two spins. ${ }^{96,97}$ Therefore, by applying electric field, the helicity $\vec{S}_{i} \times \vec{S}_{j}$ can in principle be switched.

Even more exotic topological phenomena will be the research targets in the long run. One is the quantized anomalous Hall effect theoretically predicted to occur in two-dimensional metallic 
ferromagnets, provided that the Hall conductance is larger than $\frac{e^{2}}{2 h}$ while the diagonal conductance is small on this scale. ${ }^{98}$ Oxide interface electrons are promising candidates to realize these conditions with careful design of the carrier density and mobility, and the band structure hosting the strong SOI. The other example is the quantum spin Hall system/topological insulator, which is characterized by the nontrivial topological structure of the Bloch wavefunctions in momentum space. ${ }^{99,100}$ An interesting direction to pursue in this respect is the interplay between the topology and the electron correlation, for which the electrons at oxide interfaces are promising candidates. A related theoretical proposal has been made for a quasi-two dimensional Ir-oxide with honeycomb lattice structure. ${ }^{101}$

Finally, we should remark on recent or near-future advances in the experimental means to both prepare and probe the interface electronic state. Underlying these and many related investigations is a direct confrontation of materials chemistry on a microscopic scale. While the structures described here are meta-stable, they further require the simultaneous stabilization of components which can require vastly different oxygen potentials in their bulk synthesis. Furthermore, the requirements for interface abruptness are extremely challenging. A variety of state of the art probes of the local electronic structure (electron energy loss spectroscopy, ${ }^{10}$ resonant $\mathrm{x}$-ray scattering, ${ }^{102}$ tomography, ${ }^{103}$ etc.) generically find that the length-scale for charge transfer is $\sim 1 \mathrm{~nm}$. Thus probing this feature in the intrinsic regime essentially requires atomicscale control. As for detailed measurements of the electronic structure, angle-resolved photoemission spectroscopy (ARPES) has been the principal probe of the quasi-two-dimensional electronic state for these decades, and is now evolving into a more bulk-sensitive tool with use of excitations of lower-energy (several-eV laser light) or higher-energy (hard x-ray) light. Thus, ARPES is now applicable to buried interfaces beneath the surface, as well as resonant soft and hard x-ray scattering and absorption spectroscopy. In particular, soft x-ray reflectometry with variable photon energy and polarization can yield atomic layer-resolved profiles of the charge, ${ }^{104}$ spin, $^{35}$ and orbital ${ }^{46}$ polarization in oxide heterostructures and superlattices. Other noteworthy advances for potential probes is in spin-polarized neutron reflectometry, detecting the interface magnetic state, ${ }^{105}$ and the ultra-slow positive muon probe attaining nanometer depth-profile resolution; ${ }^{106}$ both will be reinforced by emerging pulsed spallation sources. In-situ control experiments, for example tracing the change of the interface state while exciting the gate electric field or current, will be of increasing importance to reveal such emergent properties of oxide interfaces.

To conclude we have discussed the science of oxide interfaces from the viewpoint of emergent phenomena due to strong electron correlations. Combining advances in experimental techniques and new concepts supported by first-principles electronic structure calculations, this system will be an ideal arena for physics, chemistry, and also technology in the years to come. 


\section{ACKNOWLEDGEMENTS}

This work was partly supported by the Japan Society for the Promotion of Science (JSPS) through its Funding Program for World-Leading Innovative R\&D on Science and Technology (FIRST Program). H.Y.H. acknowledges support from the Department of Energy, Office of Basic Energy Sciences, under contract DE-AC02-76SF00515. B.K. acknowledges support by the German Science Foundation under collaborative Grant No. SFB/TRR80. N.N. acknowledges support by MEXT Grand-in-Aid No. 20740167, 19048008, 19048015, 21244053, and the Strategic International Cooperative Program (Joint Research Type) from the Japan Science and Technology Agency.

\section{REFERENCES}

1 Imada, M., Fujimori, A. \& Tokura, Y. Metal-insulator transitions. Rev. Mod. Phys. 70, 10391262 (1998).

2 Heber, J. Enter the oxides. Nature 459, 28-30 (2009).

3 Anderson, P. W. More is different. Science 177, 393-396 (1972).

4 Bychkov, Y. A. \& Rashba, E. I. Properties of a 2D electron gas with lifted spectral degeneracy. JETP Lett. 39, 78-81 (1984).

5 Oka, T. \& Nagaosa, N. Interfaces of correlated electron systems: proposed mechanism for colossal electroresistance. Phys. Rev. Lett. 95, 266403 (2005). Takahashi, K. S., Kawasaki, M. \& Tokura, Y. Interface ferromagnetism in oxide superlattices of $\mathrm{CaMnO}_{3} / \mathrm{CaRuO}_{3}$. Appl. Phys. Lett. 79, 1324-1326 (2001).

7 Goodenough, J. B. Theory of the role of covalence in the perovskite-type manganites [La, M(II)] $\mathrm{MnO}_{3}$. Phys. Rev. 100, 564-573 (1955).

8 Goodenough, J. B. An interpretation of the magnetic properties of the perovskite-type mixed crystals $\mathrm{La}_{1-\mathrm{x}} \mathrm{Sr}_{\mathrm{x}} \mathrm{CoO}_{3-\lambda}$. J. Phys. Chem. Solids 6, 287-297 (1958).

9 Kanamori, J. Superexchange interaction and symmetry properties of electron orbitals. J. Phys. Chem. Solids 10, 87-98 (1959).

10 Ohtomo, A., Muller, D. A., Grazul, J. L. \& Hwang, H. Y. Artificial charge-modulation in atomic-scale perovskite titanate superlattices. Nature 419, 378-380 (2002).

11 Tokura, Y. et al. Filling dependence of electronic properties on the verge of metal-Mottinsulator transitions in $\mathrm{Sr}_{1-x} \mathrm{La}_{\mathrm{x}} \mathrm{TiO}_{3}$. Phys. Rev. Lett. 72, 2126-2129 (1993).

12 Salvador, P. A., Haghiri-Gosnet, A.-M., Mercey, B., Hervieu, M. \& Raveau, B. Growth and magnetoresistive properties of $\left(\mathrm{LaMnO}_{3}\right)_{\mathrm{m}}\left(\mathrm{SrMnO}_{3}\right)_{\mathrm{n}}$ superlattices. Appl. Phys. Lett. 75, 26382640 (1999).

13 Yamada, H., Kawasaki, M., Lottermoser, T., Arima, T. \& Tokura, Y. LaMnO $3 / \mathrm{SrMnO}_{3}$ interfaces with coupled charge-spin-orbital modulation. Appl. Phys. Lett. 80, 52506 (2006). 
14 Bhattacharya, A. et al. The metal-insulator transition and its relation to magnetic structure in $\left(\mathrm{LaMnO}_{3}\right)_{2 \mathrm{n}} /\left(\mathrm{SrMnO}_{3}\right)_{\mathrm{n}}$ superlattices. Phys. Rev. Lett. 100, 257203 (2008).

15 Gozar, A. et al. High-temperature interface superconductivity between metallic and insulating copper oxides. Nature 455, 782-785 (2008).

16 Yuli, O. et al. Enhancement of the superconducting transition temperature of $\mathrm{La}_{2-\mathrm{x}} \mathrm{Sr}_{\mathrm{x}} \mathrm{CuO}_{4}$ bilayers: role of pairing and phase stiffness. Phys. Rev. Lett. 101, 057005 (2008).

17 Berg, E., Orgad, D. \& Kivelson S. A. Route to high-temperature superconductivity in composite systems. Phys. Rev. B 78, 094509 (2008).

18 Okamoto, S. \& Maier, T. A. Enhanced superconductivity in superlattices of high- $\mathrm{T}_{\mathrm{C}}$ cuprates. Phys. Rev. Lett. 101, 156401 (2008).

19 Ohtomo, A. \& Hwang, H. Y. A high-mobility electron gas at the $\mathrm{LaAlO}_{3} / \mathrm{SrTiO}_{3}$ heterointerface. Nature 427, 423-426 (2004).

${ }^{20}$ Baraff, G. A., Appelbaum, J. A. \& Hamann, D. R. Self-consistent calculation of the electronic structure at an abrupt GaAs-Ge interface. Phys. Rev. Lett. 38, 237-240 (1977).

21 Harrison, W. A., Kraut, E. A., Waldrop, J. R. \& Grant, R.W. Polar heterojunction interfaces. Phys. Rev. B 18, 4402-4410 (1978).

22 Nakagawa, N., Hwang, H. Y. \& Muller, D. A. Why some interfaces cannot be sharp. Nature Mater. 5, 204-209 (2006).

${ }^{23}$ Hesper, R., Tjeng, L. H., Heeres, A. \& Sawatzky, G. A. Photoemission evidence of electronic stabilization of polar surfaces in $\mathrm{K}_{3} \mathrm{C}_{60}$. Phys. Rev. B 62, 16046-16055 (2000).

24 Stengel, M. \& Vanderbilt, D. Berry-phase theory of polar discontinuities at oxide-oxide interfaces. Phys. Rev. B 80, 241103(R) (2009).

${ }^{25}$ Kalabukhov, A. et al. Effect of oxygen vacancies in the $\mathrm{SrTiO}_{3}$ substrate on the electrical properties of the $\mathrm{LaAlO}_{3} / \mathrm{SrTiO}_{3}$ interface. Phys. Rev. B 75, 121404(R) (2007).

26 Siemons, W. et al. Origin of charge density at $\mathrm{LaAlO}_{3}$ on $\mathrm{SrTiO}_{3}$ heterointerfaces: possibility of intrinsic doping. Phys. Rev. Lett. 98, 196802 (2007).

27 Herranz, G. et al. High mobility in $\mathrm{LaAlO}_{3} / \mathrm{SrTiO}_{3}$ heterostructures: origin, dimensionality, and perspectives. Phys. Rev. Lett. 98, 216803 (2007).

28 Brinkman, A. et al. Magnetic effects at the interface between non-magnetic oxides. Nature Mater. 6, 493-496 (2007).

${ }^{29}$ Cen, C. et al. Nanoscale control of an interfacial metal-insulator transition at room temperature. Nature Mater. 7, 298-302 (2008).

30 Xie, Y. W., Bell, C., Yajima, T., Hikita, Y. \& Hwang, H. Y. Charge writing at the $\mathrm{LaAlO}_{3} / \mathrm{SrTiO}_{3}$ surface. Nano Lett. 10, 2588-2591 (2010).

31 Hotta, Y., Susaki, T. \& Hwang, H. Y. Polar discontinuity doping of the $\mathrm{LaVO}_{3} / \mathrm{SrTiO}_{3}$ interface. Phys. Rev. Lett. 99, 236805 (2007).

32 Higuchi, T., Hotta, Y., Susaki, T., Fujimori, A. \& Hwang, H. Y. Modulation doping of a Mott quantum well by a proximate polar discontinuity. Phys. Rev. B 79, 075415 (2009).

33 Lanier, C. H. et al. Surface reconstruction with a fractional hole: $(\sqrt{ } 5 \times \sqrt{ } 5) R 26.6^{\circ} \mathrm{LaAlO}_{3}$ (001). Phys. Rev. Lett. 98, 086102 (2007). 
34 Takizawa, M. et al. Spectroscopic evidence for competing reconstructions in polar multilayers $\mathrm{LaAlO}_{3} / \mathrm{LaVO}_{3} / \mathrm{LaAlO}_{3}$. Phys. Rev. Lett. 102, 236401 (2009).

35 Freeland, J. W. et al. Charge transport and magnetization profile at the interface between the correlated metal $\mathrm{CaRuO}_{3}$ and the antiferromagnetic insulator $\mathrm{CaMnO}_{3}$. Phys. Rev. B 81, 094414 (2010).

${ }^{36}$ Pauli, S. A. et al. Evolution of the interfacial structure of $\mathrm{LaAlO}_{3}$ on $\mathrm{SrTiO}_{3}$. Phys. Rev. Lett. 106, 036101 (2011).

37 Salluzzo, M. et al. Orbital reconstruction and the two-dimensional electron gas at the $\mathrm{LaAlO}_{3} / \mathrm{SrTiO}_{3}$ interface. Phys. Rev. Lett. 102, 166804 (2009).

38 Pinta, C. et al. Suppression of spin-state transition in epitaxially strained $\mathrm{LaCoO}_{3}$. Phys. Rev. B 78, 174402 (2008).

39 Aruta, C. et al. Orbital occupation, atomic moments, and magnetic ordering at interfaces of manganite thin films. Phys. Rev. B 80, 014431 (2009).

40 Okamoto, S. \& Millis, A. J. Electronic reconstruction at an interface between a Mott insulator and a band insulator. Nature 428, 630-633 (2004).

${ }^{41}$ Pentcheva, R. \& Pickett, W. E. Correlation-driven charge order at the interface between a Mott and a band insulator. Phys. Rev. Lett. 99, 016802 (2007).

42 Chaloupka, J. \& Khaliullin, G. Orbital order and possible superconductivity in $\mathrm{LaNiO}_{3} / \mathrm{LaMO}_{3}$ superlattices. Phys. Rev. Lett. 100, 016404 (2008).

43 Hansmann, P. et al. Turning a nickelate Fermi surface into a cuprate-like one through heterostructuring. Phys. Rev. Lett. 103, 016401 (2009).

${ }^{44}$ Han, M. J., Marianetti, C. A. \& Millis, A. J. Chemical control of orbital polarization in artificially structured transition-metal oxides: $\mathrm{La}_{2} \mathrm{NiXO}_{6} \quad(\mathrm{X}=\mathrm{B}, \mathrm{Al}, \mathrm{Ga}, \mathrm{In})$ from first principles. Phys. Rev. B 82, 134408 (2010).

45 Seo, S. S. A. et al. Two-dimensional confinement of $3 d^{1}$ electrons in $\mathrm{LaTiO}_{3}-\mathrm{LaAlO}_{3}$ multilayers. Phys. Rev. Lett. 104, 036401 (2010).

46 Benckiser, E. et al. Orbital reflectometry of oxide heterostructures. Nature Mater. 10, 189-193 (2011).

47 Chakhalian, J. et al. Magnetism at the interface between ferromagnetic and superconducting oxides. Nature Phys. 2, 244-248 (2006).

${ }^{48}$ Chakhalian, J. et al. Orbital reconstruction and covalent bonding at an oxide interface. Science 318, 1114-1117 (2007).

$49 \mathrm{Yu}$, P. et al. Interface ferromagnetism and orbital reconstruction in $\mathrm{BiFeO}_{3}-\mathrm{La}_{0.7} \mathrm{Ca}_{0.3} \mathrm{MnO}_{3}$ heterostructures. Phys. Rev. Lett. 105, 027201 (2010).

50 Jackeli, G. \& Khaliullin, G. Spin, charge, and orbital order at the interface between correlated oxides. Phys. Rev. Lett. 101, 216804 (2008).

51 Sai, N., Meyer, B. \& Vanderbilt, D. Compositional inversion symmetry breaking in ferroelectric perovskites. Phys. Rev. Lett. 84, 5636-5639 (2000).

52 Warusawithana, M.P., Colla, E.V., Eckstein, J.N. \& Weissman M.B. Artificial dielectric superlattices with broken inversion symmetry. Phys. Rev. Lett. 90, 036802 (2003). 

polarization enhancement in asymmetric three-component ferroelectric superlattices. Nature 433, 395-399 (2005).

54 Yamada, H., Kawasaki, M., Ogawa, Y. \& Tokura, Y. Perovskite oxide tricolor superlattices with artificially broken inversion symmetry by interface effects. Appl. Phys. Lett. 81, 47934795 (2002).

55 Ogawa, Y. et al. Nonlinear magneto-optical Kerr rotation of an oxide superlattice with artificially broken symmetry. Phys. Rev. Lett. 90, 217403 (2003).

56 Kida, N. et al. Optical magnetoelectric effect of patterned oxide superlattices with ferromagnetic interfaces, Phys. Rev. Lett. 99, 197404 (2007).

57 Yamada, H. et al. Engineered interface of magnetic oxides. Science 305, 646-648 (2004).

58 Reyren, N. et al. Superconducting interfaces between insulating oxides. Science 317, 11961199 (2007).

59 Caviglia, A. D. et al. Electric field control of the $\mathrm{LaAlO}_{3} / \mathrm{SrTiO}_{3}$ interface ground state. Nature 456, 624-627 (2008).

60 Bell, C. et al. Dominant mobility modulation by the electric field effect at the $\mathrm{LaAlO}_{3} / \mathrm{SrTiO}_{3}$ interface. Phys. Rev. Lett. 103, 226802 (2009).

61 Schooley, J. F. et al. Dependence of the superconducting transition temperature on carrier concentration in semiconducting $\mathrm{SrTiO}_{3}$. Phys. Rev. Lett. 14, 305-307 (1965).

62 Ahn, C. H., Triscone, J.-M. \& Mannhart, J. Electric field effect in correlated oxide systems. Nature 424, 1015-1018 (2003).

63 Ueno, K. et al. Electric-field-induced superconductivity in an insulator. Nat. Mater. 7, 855858 (2008).

64 Shimotani, H. et al. Insulator-to-metal transition in ZnO by electric double layer gating. Appl. Phys. Lett. 91, 082106 (2007).

65 Yuan, H. T. et al. High-density carrier accumulation in $\mathrm{ZnO}$ field-effect transistors gated by electric double layers of ionic liquids. Adv. Fun. Mater. 19, 1046-1053 (2009).

${ }^{66}$ Ye, J. T. et al. Gate-induced interface superconductivity on an atomically flat film. Nat. Mater. 9, 125-128 (2010).

67 Dhoot, A. S. et al. Increased $\mathrm{T}_{\mathrm{c}}$ in electrolyte-gated cuprates. Adv. Mater. 22, 2529-2533 (2010).

${ }^{68}$ Kozuka, Y. et al. Enhancing the electron mobility via delta-doping in $\mathrm{SrTiO}_{3}$. Appl. Phys. Lett. 97, 222115 (2010).

69 Jalan, B., Stemmer, S., Mack, S. \& Allen, S. J. Two-dimensional electron gas in $\delta$-doped $\mathrm{SrTiO}_{3}$. Phys. Rev. B 82, 081103 (2010).

${ }^{70}$ Kozuka, Y. et al. Two-dimensional normal-state quantum oscillations in a superconducting heterostructure. Nature 462, 487-490 (2009).

${ }^{71} \mathrm{Kim}$, M. et al. Fermi surface and superconductivity in low-density high-mobility $\delta$-doped $\mathrm{SrTiO}_{3}$. arXiv: 1104.3388 (Phys. Rev. Lett., in press). 
${ }^{72}$ Caviglia, A. D. et al. Tunable Rashba spin-orbit interaction at oxide interfaces. Phys. Rev. Lett. 104, 126803 (2010).

${ }^{73}$ Shalom, M. B. et al. Tuning spin-orbit coupling and superconductivity at the $\mathrm{SrTiO}_{3} / \mathrm{LaAlO}_{3}$ interface: a magnetotransport study. Phys. Rev. Lett. 104, 126802 (2010).

${ }^{74}$ Dikin, D. A. et al. Coexistence of superconductivity and ferromagnetism in two dimensions. Phys. Rev. Lett. 107, 056802 (2011).

${ }^{75}$ Li, L., Richter, C., Mannhart, J. \& Ashoori, R. C. Coexistence of magnetic order and twodimensional superconductivity at $\mathrm{LaAlO}_{3} / \mathrm{SrTiO}_{3}$ interfaces. arXiv: 1105.0235 (Nature Phys., in press).

${ }^{76}$ Bert, J. A. et al. Direct imaging of the coexistence of ferromagnetism and superconductivity at the $\mathrm{LaAlO}_{3} / \mathrm{SrTiO}_{3}$ interface. arXiv: 1108.3150 (Nature Phys., in press).

${ }^{77}$ Michaeli, K., Potter, A. C. \& Lee, P. A. Superconductivity and ferromagnetism in oxide interface structures: possibility of finite momentum pairing. arXiv: 1107.4352.

${ }^{78}$ Stormer, H. L. Nobel Lecture: the fractional quantum Hall effect. Rev. Mod. Phys. 71, 875889 (1999).

${ }^{79}$ Tokura, Y. \& Hwang, H. Y. Complex oxides on fire. Nature Mater. 7, 694-695 (2008).

${ }^{80}$ Tsukazaki, A. et al. Quantum Hall effect in polar oxide heterostructures. Science 315, 13881391 (2007).

81 Tsukazaki, A. et al. Observation of the fractional quantum Hall effect in an oxide. Nature Mater. 9, 889-893 (2010).

${ }^{82}$ Schlom, D. G. \& Pfeiffer L. N. Upward mobility rocks! Nature Mater. 9, 881-883 (2010).

${ }^{83}$ Kozuka, Y. et al. Insulating phase of a two-dimensional electron gas in $\mathrm{Mg}_{x} \mathrm{Zn}_{1-x} \mathrm{O} / \mathrm{ZnO}$ heterostructures below $v=1 / 3$. Phys. Rev. B 84, 033304 (2011).

${ }^{84}$ Tanatar, B. \& Ceperley, D. Ground state of the two-dimensional electron gas. Phys. Rev. B 39, 5005 -5016 (1989).

${ }^{85}$ Tsukazaki, A. et al. Spin susceptibility and effective mass of two-dimensional electrons in $\mathrm{Mg}_{x} \mathrm{Zn}_{1-x} / \mathrm{ZnO}$ heterostructures. Phys. Rev. B 78, 233308 (2008).

${ }^{86}$ De Poortere, E. P., Tutuc, E., Papadakis, S. J. \& Shayegan, M. Resistance spikes at transitions between quantum Hall ferromagnets. Science 290, 1546-1549 (2000).

${ }^{87}$ Kroemer, H. Quasi-electric fields and band offsets: teaching electrons new tricks. Nobel Lecture, December 8, 2000.

${ }^{88}$ Waser, R. \& Aono, M. Nanoionics-based resistive switching memories. Nature Mater. 6, 833840 (2007).

${ }^{89}$ Sawa, A. Resistive switching in transition metal oxides. Mater. Today 11, 28-36 (2008).

${ }^{90}$ Nakamura, M., Sawa, A., Fujioka, J., Kawasaki, M. \& Tokura, Y. Interface band profiles of Mott-insulator/Nb:SrTiO 3 heterojunctions as investigated by optical spectroscopy. Phys. Rev. B 82, 201101 (2010).

${ }^{91}$ Matsubara, M. et al. Photoinduced switching between charge and orbital ordered insulator and ferromagnetic metal in perovskite manganites. Phys. Rev. B 77, 094410 (2008). 
${ }^{92} \mathrm{Kim}, \mathrm{B}$. J. et al. Novel $\mathrm{J}_{\mathrm{eff}}=1 / 2$ Mott state induced by relativistic spin-orbit coupling in $\mathrm{Sr}_{2} \mathrm{IrO}_{4}$. Phys. Rev. Lett. 101, 076402 (2008).

93 Datta, S. \& Das B. Electronic analog of the electro-optic modulator. Appl. Phys. Lett. 56, 665667 (1990).

94 Chernyshov, A. et al. Evidence for reversible control of magnetization in a ferromagnetic material by means of spin-orbit magnetic field. Nature Phys. 5, 656-659 (2009).

95 Miron, I. M. et al. Current-driven spin torque induced by the Rashba effect in a ferromagnetic metal layer. Nature Mater. 9, 230-234 (2010).

96 Kimura, T. et al. Magnetic control of ferroelectric polarization. Nature 426, 55-58 (2003).

97 Katsura, H., Nagaosa, N. \& Balatsky, A.V. Spin current and magnetoelectric effect in noncollinear magnets. Phys. Rev. Lett. 95, 057205 (2005).

98 Onoda, M. \& Nagaosa, N. Quantized anomalous Hall effect in two-dimensional ferromagnets: quantum Hall effect in metals. Phys. Rev. Lett. 90, 206601 (2003).

99 Hasan, M. Z. \& Kane, C. L. Topological insulators. Rev. Mod. Phys. 82, 3045-3067 (2010).

${ }^{100}$ Qi, X. L. \& Zhang, S. C. Topological insulators and superconductors. arXiv:1008.2026 (Rev. Mod. Phys., in press).

101 Shitade, A. et al. Quantum spin Hall effect in a transition metal oxide $\mathrm{Na}_{2} \mathrm{IrO}_{3}$. Phys. Rev. Lett. 102, 256403 (2009).

${ }^{102}$ Smadici, S. et al. Electronic reconstruction at $\mathrm{SrMnO}_{3}-\mathrm{LaMnO}_{3}$ superlattice interfaces. Phys. Rev. Lett. 99, 196404 (2007).

${ }^{103}$ Logvenov, G., Gozar, A. \& Bozovic, I. High-temperature superconductivity in a single copper-oxygen plane. Science 326, 699-702 (2009).

${ }^{104}$ Smadici, S. et al. Superconducting transition at $38 \mathrm{~K}$ in insulating-overdoped $\mathrm{La}_{2} \mathrm{CuO}_{4}$ $\mathrm{La}_{1.64} \mathrm{Sr}_{0.36} \mathrm{CuO}_{4}$ superlattices: evidence for interface electronic redistribution from resonant soft x-Ray scattering. Phys. Rev. Lett. 102, 107004 (2009).

105 May, S. J. et al. Magnetically asymmetric interfaces in a $\left(\mathrm{LaMnO}_{3}\right) /\left(\mathrm{SrMnO}_{3}\right)$ superlattice due to structural asymmetries. Phys. Rev. B 77, 174409 (2008).

${ }^{106}$ Boris A. V. et al. Dimensionality control of electronic phase transitions in nickel-oxide superlattices. Science 332, 937-940 (2011). 